\title{
Optimal filtering with linear canonical transformations
}

\author{
Billur Barshan, M. Alper Kutay, Haldun M. Ozaktas \\ Department of Electrical Engineering, Bilkent University, Bilkent, 06533 Ankara, Turkey
}

Received 25 June 1996; accepted 12 September 1996

\begin{abstract}
Optimal filtering with linear canonical transformations allows smaller mean-square errors in restoring signals degraded by linear time- or space-variant distortions and non-stationary noise. This reduction in error comes at no additional computational cost. This is made possible by the additional flexibility that comes with the three free parameters of linear canonical transformations, as opposed to the fractional Fourier transform which has only one free parameter, and the ordinary Fourier transform which has none. Application of the method to severely degraded images is shown to be significantly superior to filtering in fractional Fourier domains in certain cases.
\end{abstract}

\section{Introduction}

In this paper, we consider a signal observation model of the form

$g(t)=\int_{-\infty}^{+\infty} h\left(t, t^{\prime}\right) f\left(t^{\prime}\right) \mathrm{d} t^{\prime}+n(t)$,

where $g(t)$ is the observed signal, $f(t)$ is the signal we wish to recover, $n(t)$ is an additive and possibly non-stationary noise signal, and $h\left(t, t^{\prime}\right)$ is the kernel representing an undesired time-varying linear distortion.

A general linear estimate $\hat{f}(t)$ of $f(t)$ in terms of $g(t)$ may be expressed as

$\hat{f}(t)=\int_{-\infty}^{+\infty} k\left(t, t^{\prime}\right) g\left(t^{\prime}\right) \mathrm{d} t^{\prime}$,

where $k\left(t, t^{\prime}\right)$ is the kernel representing the recovery operation.

Under a number of assumptions and given the relevant correlation functions, the optimal kernel $k_{\text {opt }}\left(t, t^{\prime}\right)$, which minimizes the mean-square error

$$
\begin{aligned}
\sigma^{2} & \equiv E\left\{\|f-\hat{f}\|^{2}\right\} \\
& \equiv E\left\{\int_{-\infty}^{+\infty}[f(t)-\hat{f}(t)]^{*}[f(t)-\hat{f}(t)] \mathrm{d} t\right\},
\end{aligned}
$$

is well known [1], and will be reviewed below. However, obtaining the most general linear estimate by using Eq. (2) requires computational time of the order of $N^{2}$, where $N$ is the time- or space-bandwidth product of the signals. Under some conditions, it is possible to obtain satisfactory estimates in much shorter time. For instance, when the signals involved are stationary and $h\left(t, t^{\prime}\right)$ is a time-invariant kernel, the optimal kernel $k_{\text {opt }}\left(t, t^{\prime}\right)$ turns out to be a time-invariant (or space-invariant) filter which can be implemented in $\mathscr{O}(N \log N)$ time. This solution, which corresponds to multiplicative filtering in the Fourier domain, is known as the classical time-invariant Wiener filter [2].

In Refs. [3,4], the authors show that filtering in fractional Fourier domains $[5,6]$ can offer significant improvement with respect to filtering in the ordinary Fourier domain for particular signal, noise and distortion characteristics. This approach allows smaller mean-square errors to be achieved while keeping the time of computation at $\mathscr{O}(N \log N)$. These improvements are made possible by the additional flexibility afforded by the fractional order parameter.

Here, we provide a generalization of the concept of filtering in fractional Fourier domains which provides even greater flexibility and thus even smaller errors in certain cases, while still maintaining $\mathscr{O}(N \log N)$ computational time. Thus, the benefits achieved come without any additional cost. Our method is based on linear canonical transformations which are a family of transformations with three parameters [7]. (The fractional Fourier transform is a special case of the linear canonical transformations with 
only a single parameter.) These transformations can be computed using a fast algorithm in $\mathscr{O}(N \log N)$ time. We first review the classical problem so as to provide a proper context and establish the necessary notation. Then we consider recovery filters which correspond to multiplication by a function in the transform domain. The optimum multiplicative function will be derived analytically for a given transform domain characterized by the three parameters of the linear canonical transformation employed. Subsequently, we will seek the optimal values of these parameters, thus achieving the smallest possible error with the proposed method.

\section{The classical problem}

In this section, the most general linear filtering problem will be briefly reviewed. Assumptions on the signal and noise statistics are as follows: The noise process is zeromean and independent of the signal process. Both processes are, in general, non-stationary and their covariance functions $R_{f f}\left(t, t^{\prime}\right)$ and $R_{n n}\left(t, t^{\prime}\right)$ are known. Under these assumptions, the cross-correlation function $R_{f g}\left(t, t^{\prime}\right)$ between the input process $f(t)$ and the output process $g(t)$, and the correlation function $R_{g g}\left(t, t^{\prime}\right)$ of the output process are found as

$R_{f g}\left(t, t^{\prime}\right)=\int_{-\infty}^{+\infty} h\left(t, t^{\prime}\right) R_{f f}\left(t, t^{\prime \prime}\right) \mathrm{d} t^{\prime \prime}$,

$R_{g g}\left(t, t^{\prime}\right)=\int_{-\infty}^{+\infty} h^{*}\left(t, t^{\prime}\right) R_{f g}\left(t, t^{\prime \prime}\right) \mathrm{d} t^{\prime \prime}+R_{n n}\left(t, t^{\prime}\right)$.

The most general optimal linear filter with kernel $k_{\text {opt }}\left(t, t^{\prime}\right)$ enables us to obtain an estimate

$\hat{f}(t)=\int_{-\infty}^{+\infty} k_{\text {opt }}\left(t, t^{\prime}\right) g\left(t^{\prime}\right) \mathrm{d} t^{\prime}$,

such that the mean-square error given by

$\sigma^{2} \equiv E\left\{\|f(t)-\hat{f}(t)\|^{2}\right\}$,

is minimized. Here, $\|\cdot\|$ denotes the $L_{2}$ norm:

$\|f(t)\|^{2}=\int_{\infty}^{+\infty} f(t)^{*} f(t) \mathrm{d} t$.

The above definition of the mean-square error is appropriate for non-stationary signals of finite energy, whose functional representations are square integrable. It is known that the optimal kernel satisfies the following equality known as the Wiener-Hopf equation [1]:

$R_{f g}\left(t, t^{\prime}\right)=\int_{-\infty}^{+\infty} k_{\mathrm{opt}}\left(t, t^{\prime}\right) R_{g g}\left(t, t^{\prime \prime}\right) \mathrm{d} t^{\prime \prime} \quad \forall t, t^{\prime}$.

As we have mentioned before, obtaining an estimate by using Eq. (6) requires $\mathscr{Q}\left(N^{2}\right)$ computation time.

\section{Filtering with linear canonical transformations}

The class of linear canonical transformations is defined as

$f_{p}\left(t_{p}\right)=\mathscr{Q}_{p}[f(t)]\left(t_{p}\right)=\int_{-\infty}^{+\infty} Q_{p}\left(t_{p}, t\right) f(t) \mathrm{d} t$,

with

$Q_{p}\left(t_{p}, t\right)=C_{p} \exp \left[i \pi\left(\alpha t_{p}^{2}-2 \beta t_{p} t+\gamma t^{2}\right)\right]$,

$C_{p}=\beta^{1 / 2} \exp (-i \pi / 4)$,

where we introduced the parameter vector $p=[\alpha \beta \gamma]$ with $\alpha, \beta$, and $\gamma$ being the three real parameters characterizing the transformation. All members of this class of transformations are unitary. The signal $f_{p}\left(t_{p}\right)$ will be referred to as the transform domain representation of $f(t)$. When $\alpha=\gamma=0$ and $\beta=1$, the above reduces to the ordinary Fourier transform except for the inconsequential phase factor $\exp (-i \pi / 4)$. When $\alpha=\gamma=\cot (a \pi / 2)$ and $\beta=\csc (a \pi / 2)$, the above reduces to the ath order fractional Fourier transform [9-13], again except for a phase factor.

We will seek estimators of the form

$\hat{f}(t)=\mathscr{Q}_{p}^{-1}\left[m\left(t_{p}\right) \mathscr{Q}_{p}[g(t)]\left(t_{p}\right)\right](t)$,

where $\mathscr{Q}_{p}$ is the linear canonical transformation operator and $m\left(t_{p}\right)$ is a multiplicative filter applied in the transform domain. According to the above equation, first the linear canonical transform of the signal is taken with parameters $\alpha, \beta$ and $\gamma$. Then, the transformed signal is multiplied with the filter $m\left(t_{p}\right)$, and finally, the inverse linear canonical transform of the resulting signal is taken. In the following, the optimal filter function in a given transform domain will be derived for a given parameter vector and then the mean-square error will be minimized over the parameter vector.

The set of linear filters which can be realized in this manner is a subset of the class of general linear filters. Thus, the optimal filter we obtain will not be the most optimal among all linear filters. However, the class of filters we consider is a much broader class than ordinary Fourier and fractional Fourier domain filters. In certain situations involving time- or space-varying degradation models and non-stationary processes, it is possible to obtain smaller mean-square errors in comparison to filtering in the conventional Fourier domain or the fractional Fourier domain. This reduction in mean-square error comes at no additional cost because the resulting filter can be implemented in $\mathscr{O}(N \log N)$ time [8] just like the ordinary Fourier transform. It can also be implemented optically with a setup similar to that used for implementing the ordinary Fourier transform [14-16]. 
Since $\mathscr{Q}_{p}$ is a unitary transform, the mean-square error is the same in the Iransforn domain:

$\sigma_{p}^{2} \equiv E\left\{\|f(t)-\hat{f}(t)\|^{2}\right\}=E\left\{\left\|f_{p}\left(t_{p}\right)-\hat{f}_{p}\left(t_{p}\right)\right\|^{2}\right\}$.

Now, by inserting

$\hat{f}_{p}\left(t_{p}\right)=\mathscr{Q}_{p}[\hat{f}(t)]\left(t_{p}\right)=\left[m\left(t_{p}\right) \mathscr{Q}_{p}[g(t)]\left(t_{p}\right)\right](t)$

in the rightmost expression of the above equation, and minimizing $\sigma_{p}^{2}$ with respect to $m\left(t_{p}\right)$, yields the following result

$E\left\{\left[f_{p}\left(t_{p}\right)-\hat{f}\left(t_{p}\right)\right]^{*} g_{p}\left(t_{p}\right)\right\}=0$,

which we recognize as the orthogonality condition $[17,18]$ : According to the above condition, the best linear meansquare error estimate $\hat{f}_{p}\left(t_{p}\right)$ is an orthogonal projection of the signal $f_{p}\left(t_{p}\right)$ into the space spanned by the observations. The resulting error, which is unbiased ( $E\left\{f_{p}\left(t_{p}\right)-\right.$ $\left.\hat{f}_{p}\left(t_{p}\right)\right\}=0$ ), is orthogonal to the observation space, hence, uncorrelated with the observations. Eq. (15) can be solved for the optimum filter function $m_{\text {opt }}(\cdot)$ by using the definition of $\hat{f}_{p}^{*}\left(t_{p}\right)$, and by taking the complex conjugate of both sides of the equation

$m_{\text {opt }}\left(t_{p}\right)=\frac{R_{f_{p} g_{p}}\left(t_{p}, t_{p}\right)}{R_{g_{p} g_{p}}\left(t_{p}, t_{p}\right)}$,

where the correlation functions appearing in this equation can be obtained from the correlation functions $R_{f g}\left(t, t^{\prime}\right)$ and $R_{g g}\left(t, t^{\prime}\right)$ by

$$
\begin{aligned}
& R_{f_{p} g_{p}}\left(t_{p}, t_{p}\right) \\
& \quad=\int_{-\infty}^{+\infty} \int_{-\infty}^{+\infty} Q_{p}\left(t_{p}, t\right) Q_{p}^{-1}\left(t_{p}, t^{\prime}\right) R_{f g}\left(t, t^{\prime}\right) \mathrm{d} t^{\prime} \mathrm{d} t
\end{aligned}
$$

$$
\begin{aligned}
& R_{g_{p} g_{p}}\left(t_{p}, t_{p}\right) \\
& \quad=\int_{-\infty}^{+\infty} \int_{-\infty}^{+\infty} Q_{p}\left(t_{p}, t\right) Q_{p}^{-1}\left(t_{p}, t^{\prime}\right) R_{g g}\left(t, t^{\prime}\right) \mathrm{d} t^{\prime} \mathrm{d} t
\end{aligned}
$$

Thus, the optimal multiplicative filter function is found as:

$$
\begin{aligned}
m_{\mathrm{opt}}\left(t_{p}\right) & =\frac{\int_{-\infty}^{+\infty} \int_{-\infty}^{+\infty} Q_{p}\left(t_{p}, t\right) Q_{p}^{-1}\left(t_{p}, t^{\prime}\right) R_{f g}\left(t, t^{\prime}\right) \mathrm{d} t^{\prime} \mathrm{d} t}{\int_{-\infty}^{+\infty} \int_{-\infty}^{+\infty} Q_{p}\left(t_{p}, t\right) Q_{p}^{-1}\left(t_{p}, t^{\prime}\right) R_{g g}\left(t, t^{\prime}\right) \mathrm{d} t^{\prime} \mathrm{d} t} .
\end{aligned}
$$

By substituting the above expression in Eq. (14), we obtain an expression for the minimum mean-square error for this parameter vector:

$$
\begin{aligned}
& \sigma_{p, \mathrm{opt}}^{2} \\
& \quad=E\left\{\int_{-\infty}^{+\infty}\left[f_{p}\left(t_{p}\right)-\hat{f}_{p}\left(t_{p}\right)\right]^{*}\left[f_{p}\left(t_{p}\right)-\hat{f}_{p}\left(t_{p}\right)\right] \mathrm{d} t_{p}\right\}
\end{aligned}
$$

$$
\begin{aligned}
= & \int_{-\infty}^{+\infty}\left[R_{f_{p} \xi_{p}}\left(t_{p}, t_{p}\right)-2 \operatorname{Re}\left\{m_{\mathrm{opt}}^{*}\left(t_{p}\right) R_{f_{p} g_{p}}\left(t_{p}, t_{p}\right)\right\}\right. \\
& \left.+m_{\mathrm{opt}}\left(t_{p}\right) m_{\mathrm{opt}}^{*}\left(t_{p}\right) R_{g_{p} g_{p}}\left(t_{p}, t_{p}\right)\right] \mathrm{d} t_{p} .
\end{aligned}
$$

Now, by employing a standard multi-variate optimization routine [19], the optimal value $p_{\mathrm{opt}}$ of the parameter vector $p=[\alpha \beta \gamma]$, which minimizes $\sigma_{p, \mathrm{opt}}$ can be found.

\section{Examples}

We first present a simple one-dimensional example which illustrates the process as transparently as possible. Our original signal $f(t)$, which we choose to be a simple Gaussian function, is shown in Fig. 1(a). In Fig. 1(b), the original signal is corrupted by the presence of two strong chirp waveforms. Fig. 1(c) shows the linear canonical transform of the corrupted signal $g(t)$ for the optimal parameter vector $p_{\mathrm{opt}}=[-0.211 .75]$. In this transform domain, the original signal and the interfering chirp waveforms are effectively separated due to their small degree of overlap. Transforming back to the time domain, we obtain a fairly faithful restoration of the original signal (Fig. 1(e)) with a mean-square error of less than 0.001 . (All meansquare errors in this section have been normalized by $E\left\{\|f\|^{2}\right\}$.) For comparison, in Fig. 1(d), we show the fractional Fourier transform of the corrupted signal $g(t)$ for the optimal fractional Fourier transform order 0.7. Only one of the distorting chirps can be well separated while the other continues to corrupt the signal, so that the minimum
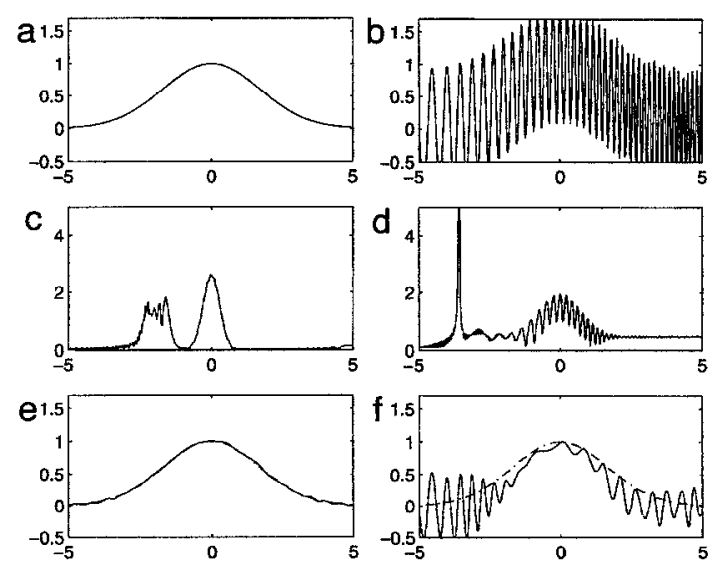

Fig. 1. (a) Original signal. (b) Corrupted signal. (c) Linear canonical transform of the corrupted signal where the interfering chirps (left) are separated from the original signal (center). (d) Fractional Fourier transform of the corrupted signal where only one of the interfering chirps (left) is separated from the original signal. (e) Signal restored by filtering in the linear canonical transform domain. (f) Signal restored by filtering in the fractional Fourier transform domain. The original signal is also shown by dashed lines for comparison. 



Fig. 2. (a) Original "Plane" image. (b) Corrupted image $(S N R \approx 1)$. (c) Image restored by filtering in the linear canonical transform domain. (d) Image restored by filtering in the fractional Fourier transform domain.


Fig. 3. (a) Original "Plane" image. (b) Corrupted image $(S N R \approx 0.1$ ). (c) Image restored by filtering in the linear canonical transform domain. (d) Image restored by filtering in the fractional Fourier transform domain. 
mean-square estimate shown in Fig. 1(e) is less than satisfactory. Note that this result is the best that can be obtained among all fractional orders, including the first order which corresponds to the ordinary Fourier transform. Thus, ordinary Fourier domain filtering would yield even less satisfactory results.

The analysis presented in this paper for the single dimension $t$ can be easily generalized to the two dimensions $x$ and $y$. Fig. 2(a) illustrates the original image used in our two-dimensional example. In Fig. 2(b), this image has been corrupted by the presence of two chirp waveforms whose amplitudes are comparable to that of the image (signal-to-noise ratio $\approx 1$ ). The optimally restored image is displayed in Fig. 2(c) for which $p_{\text {opt }}=$ [ $-0.090 .881 .57]$ and minimum mean-square error is 0.008 . For comparison, Fig. 2(d) shows the image restored in the optimal fractional Fourier domain, which we see is less satisfactory (mean-square error $=0.04$ ) than restoration in the optimal linear canonical transformation domain.

The same example is repeated with a signal-to-noise ratio $\approx 0.1$. The corresponding images are presented in Fig. 3. We see that the visual improvement offered by the linear canonical transform method (mean-square error $=$ 0.03) over the fractional Fourier method (mean-square error $=0.17)$ is greater at this lower signal-to-noise ratio.

\section{Conclusion}

We have presented a novel signal restoration method which can reduce the minimum mean-square error in comparison to ordinary or fractional Fourier domain filtering. The time complexity of this method is the same as that of ordinary Fourier-domain-based filtering. Thus the improvement obtained comes at no additional cost. This is possible because linear canonical transforms include ordinary and fractional Fourier transforms as special cases.

We have seen that images corrupted by several chirp or chirp-like waveforms whose amplitudes may be much larger than that of the original image may particularly benefit from optimal filtering with linear canonical transforms. This suggests that optimal filtering with linear canonical transforms would find many applications in optics where chirp and chirp-like distortions and noise arise naturally in optical systems, for instance, in the form of scattering from point and line defects, and twin images in holography. Also, two-dimensional linear canonical transformations and filtering are effectively and easily implemented with optical systems. Thus optics is both an application area and a means of implementation for the proposed method.
Another application arises in Synthetic Aperture Radar (SAR) which employs chirps as transmitted pulses, so that the measurements are related to the terrain reflectivity function through a chirp convolution. This process results in chirp-type disturbances caused by moving objects in the terrain, which should be removed if high resolution imaging is to be achieved [20].

\section{References}

[1] F.L. Lewis, Optimal Estimation (Wiley, New York, 1986).

[2] N. Mohanty, Signal Processing (Van Nostrand Reinhold Company, New York, 1987).

[3] M.A. Kutay, H.M. Ozaktas, L. Onural and O. Arkan, in: Proc. 1995 Int. Conf. Acoustics, Speech, and Signal Processing, IEEE, Piscataway, New Jersey (1995) pp. 937-940.

[4] M.A. Kutay, H.M. Ozaktas, O. Arkan and L. Onural, Optimal filtering in fractional Fourier domains, submitted to IEEE Trans. Signal Processing.

[5] H.M. Ozaktas, B. Barshan, D. Mendlovic and L. Onural, J. Opt. Soc. Am. A 11 (1994) 547.

[6] H.M. Ozaktas, B. Barshan and D. Mendlovic, Optical Review 1 (1994) 15.

[7] K.B. Wolf, Construction and properties of canonical transforms, in: Integral Transforms in Science and Engineering (Plenum Press, New York, 1979) chapter 9.

[8] H.M. Ozaktas, O. Arikan, M.A. Kutay and G. Bozdağ1, Digital computation of the fractional Fourier transform, submitted to IEEE Trans. Signal Processing.

[9] A.C. McBride and F.H. Kerr, IMA J. Appl. Math. 39 (1987) 159.

[10] A.W. Lohmann, J. Opt. Soc. Am. A 10 (1993) 2181.

[11] H.M. Ozaktas and D. Mendlovic, Optics Comm. 101 (1993) 163.

[12] L.B. Almeida, IEEE Trans. Signal Processing 42 (1994) 3084.

[13] H.M. Ozaktas and D. Mendlovic, J. Opt. Soc. Am. A 12 (1995) 743.

[14] M. Nazarathy and J. Shamir, J. Opt. Soc. Am. 72 (1982) 356.

[15] S. Abe and J.T. Sheridan, Optics Lett. 19 (1994) 1801.

[16] S. Abe and J.T. Sheridan, J. Phys. A 27 (1994) 4179, Corrigenda 7937.

[17] B.D.O. Anderson and J.B. Moore, Optimal Filtering (Prentice-Hall, New York, NY, 1979).

[18] A. Jazwinski, Stochastic Processes and Filtering Theory (Academic Press, New York, NY, 1970).

[19] W.H. Press, B.P. Flannery, S.A. Teukolsky and W.T. Vetterling, Numerical Recipes in Pascal (Cambridge University Press, Cambridge, UK, 1989) pp. 574-579.

[20] J.P. Fitch, Synthetic Aperture Radar (Springer-Verlag, New York, 1988) 